\title{
Curation, inference, and assessment of a globally reconstructed gene regulatory network for Streptomyces coelicolor
}

Andrea Zorro-Aranda ${ }^{1,3}$, Juan Miguel Escorcia-Rodríguez ${ }^{1}$, José Kenyi González-Kise ${ }^{1,2}$, and Julio Augusto Freyre-González ${ }^{1, *}$

${ }^{1}$ Regulatory Systems Biology Research Group, Laboratory of Systems and Synthetic Biology, and ${ }^{2}$ Undergraduate Program in Genomic Sciences, Center for Genomics Sciences, Universidad Nacional Autónoma de México. Av. Universidad s/n, Col. Chamilpa, 62210. Cuernavaca, Morelos, México. ${ }^{3}$ Bioprocess Research Group, Department of Chemical Engineering, Universidad de Antioquia, Calle 70 No. 52-21, Medellín, Colombia.

*Corresponding author: jfreyre@ccg.unam.mx (J.A.F.-G.) 


\section{Abstract}

Streptomyces coelicolor A3(2) is a model microorganism for the study of Streptomycetes, antibiotic production, and secondary metabolism in general. However, little effort to globally study its transcription has been made even though $S$. coelicolor has an outstanding variety of regulators among bacteria. We manually curated 29 years of literature and databases to assemble a metacurated experimentally-validated gene regulatory network (GRN) with 5386 genes and 9707 regulatory interactions ( $41 \%$ of the total expected interactions). This provides the most extensive and up-to-date reconstruction available for the regulatory circuitry of this organism. We found a low level of direct experimental validation for the regulatory interactions reported in the literature and curated in this work. Only $\sim 6 \%(533 / 9687)$ are supported by experiments confirming the binding of the transcription factor to the upstream region of the target gene, the so-called "strong" evidence. To tackle network incompleteness, we performed network inference using several methods (including two proposed here) for motif detection in DNA sequences and GRN inference from transcriptomics. Further, we contrasted the structural properties and functional architecture of the networks to assess the predictions' reliability, finding the inference from DNA sequence data to be the most trustworthy. Finally, we show two possible applications of the inferred and the curated network. The inferred one allowed us to identify putative novel transcription factors for the key Streptomyces antibiotic regulatory proteins (SARPs). The curated one allows us to study the conservation of the system-level components between S. coelicolor and Corynebacterium glutamicum. There we identified the basal machinery as the common signature between the two organisms. The curated networks were deposited in Abasy Atlas (https://abasy.ccg.unam.mx/) while the inferences are available as Supplementary Material.

Keywords: Streptomyces coelicolor, Gene Regulatory Networks, Network Inference, Biological Networks, Natural Decomposition Approach, Abasy Atlas

\section{Background}

Streptomycetes, the largest genus within the actinomycetes, are biotechnologically relevant organisms. They produce around half of the natural antibiotics in current use $e^{1}$. However, according to the analysis of genome mining, less than $10 \%$ of antibiotics that could be produced by actinomycetes are currently used ${ }^{2}$. Their production could be enhanced not only by experimental technologies such as genetic manipulation but also by a deeper knowledge of their secondary metabolism and transcriptional regulation. Streptomyces coelicolor A3(2) has become the model microorganism for the study of antibiotic production and secondary metabolism in general ${ }^{3}$. Before its sequencing, it was already known that S. coelicolor produces the red-pigmented antibiotic Undecylprodigiosi (RED), the blue-pigmented actinorhodin (ACT), and the calciumdependent antibiotic (CDA). However, its sequencing revealed more than 20 biosynthetic gene clusters (BGCs). Most of the metabolites produced by these clusters and their regulation are still unknown ${ }^{4}$.

S. coelicolor secondary metabolism regulation is very complex. It is controlled by a network of regulators at many levels, from global to cluster situated regulators (CSRs). Most CSRs control their own BGC, however, some of them can bind to multiple BGCs causing a cross-cluster regulation ${ }^{4}$. 
Sequencing of S. coelicolor A3(2) revealed 7825 genes, 965 of them $(\sim 12.33 \%)$ code for proteins with a predicted regulatory function. From those, 65 genes coding for sigma factors, a remarkably high number among bacteria, of which $70 \%$ (45/65) are ECF (extra-cytoplasmic function) sigma factors, suggesting independent regulation of diverse stress response regulons ${ }^{5}$. Besides, it counts with many two-component systems (TCSs), 85 sensor kinases, and 79 response regulators, also related to stress response ${ }^{5}$. The difference between sensor kinases and response regulators suggests a cross-talking among them. Noteworthy, S. coelicolor genome codes for several putative regulators that do not belong to families outside S. coelicolor ${ }^{5}$. Because of the complexity of the secondary metabolism regulation, an appropriate understanding of the S. coelicolor regulation requires it to be studied systematically at both local and global scales. At a global scale, GRN can be represented as a directed graph where nodes represent genes, and edges represent the regulatory interactions among the transcription factors (TFs) and their target genes (TGs). Previous comprehensive reviews have been focused on specific morphological differentiation and metabolic processes ${ }^{4,6-10}$. However, a GRN at a global scale is still missing.

The initial approach to reconstruct a global-scale GRN will be through text mining ${ }^{11}$. There we would be able to recollect all the information available on the literature for the microorganism. Nevertheless, it would still require manual intervention for those articles where interactions are not clearly defined. Moreover, all genes have not been studied experimentally. Therefore, alternatively, GRN inference has been applied in diverse bacteria to provide a deeper understanding of their regulatory mechanisms. Besides, it has been applied also to propose selective experimental validation of putative interactions, analyze bacterial GRN evolution, and build biological models for biotechnological processes ${ }^{12-16}$. A GRN inference for $S$. coelicolor was performed by Castro-Melchor, et. al in 2010 using ARACNE and applying module validation through the identification of consensus DNA sequences ${ }^{17}$. However, the resulting network was not assessed with any gold standard (GS) available at the time, and no thorough study of its structural properties was performed. Moreover, benchmarking studies of network inference methods have shown the poor predictive power of using a single GRN inference tool ${ }^{18}$.

Here, we performed a collection and curation of the experimentally-validated transcriptional regulatory interactions of S. coelicolor A3(2) and classified them based on the confidence level of their supporting evidence. Further, we integrated this curated GRN with previous GRNs from DBSCR (http://dbscr.hgc.jp/) and Abasy Atlas ${ }^{19}$. Then, we applied the natural decomposition approach (NDA) to identify their system-level components and unveiled different biology aspects of $S$. coelicolor regulation. Next, we applied several tools to infer novel interactions, three based on DNA binding sites for the TFs, and five based on gene expression along with two modifications proposed by the authors. We integrated the predictions using a community approach, which has been reported as the best strategy to reduce the number of false positives ${ }^{18}$. Then we used the most reliable curated network as a GS for the validation of the inferred GRNs. We further assessed the inferred networks through their structural properties and found that the NDA ${ }^{20}$ is a valuable tool for GRNs dissection and comparison. From the best-rated inferred network, we proposed new TF candidates for the direct regulation of some of the key Streptomyces antibiotic regulatory proteins (SARPS) in S. coelicolor. Finally, we applied the meta-curated network of S. coelicolor to study the conservation of the system-level components with those of its phylogenetically related 
C. glutamicum as an application of the curated network. The workflow of this work and the suggested use of the data herein reported are summarized in Figure 1.

\section{Results and discussion}

\section{Reconstruction of the most complete experimentally-validated regulatory network for S. coelicolor}

We curated a total of 124 papers retrieved from PubMed and Google Scholar queries covering a span of 29 years (from 1990 to July of 2019) (see Figure 2 and Supplementary Table 1). We collected a total of 9714 regulatory interactions (out of the 23908 expected interactions in the complete GRN as predicted by Abasy Atlas v2.4) among 5331 genes. We perceive a notable increment in the number of interactions and papers after the $S$. coelicolor genome was completely sequenced $(2002)^{5}$. This facilitates the study of its genome and regulation, being 2012 the year with most publications (see Figure 2). We classified the interactions according to their experimental evidence, expanding the RegulonDB scheme ${ }^{21,22}$. First, we label the interactions as "strong" or as "weak" according to the methodology of the experiment performed. "Strong" evidence level is assigned to experiments that prove a physical regulatory interaction between the TF and the TG. This means that the TF can bind to the upstream region of the regulated gene. Here we have experiments such as EMSA in purified proteins or in vitro transcription assays. On the other hand, "weak" evidence level is assigned when there is no evidence of direct interaction. This means the experiment suggests either a hypothetical DNA binding site, such as ChIP; or an effect of the TF over the gene that might be indirect, through another TF, such as Microarray, RNA-Seq, RT-PCR, etc. For experiments that were not in the RegulonDB scheme, such as DNA-affinity capture assay (DACA) ${ }^{23}$, we analyzed their methodology to classify them either as "strong" or "weak" evidence. In Supplementary File 2, you can find the evidence classification for each interaction according to their "strongest" supporting experiment (Supplementary Table 1 and 2).

Afterward, we gathered these interactions along with others from the databases, RegTransBase ${ }^{24}$ available at Abasy Atlas database (https://abasy.ccg.unam.mx) ${ }^{19}$, and DBSCR (http://dbscr.hgc.jp/). We processed these curated interactions to construct the corresponding GRNs, removing redundancy by mapping the gene identifiers to locus tags and merging interactions while preserving the information about the effect and evidence classification of the supporting experiments, as previously reported ${ }^{19}$. From our curation, we reconstructed a total of seven curated networks with different evidence classification and completeness. 1) Curated_FL with a total of 9454 unique interactions, from which 5\% (493/9454) are "strong". 2) Curated_FL(CS) with the 493 "strong" interactions from Curated_FL. 3) Curated_DBSCR with the 341 interactions from DBSCR and used the $\sim 34 \%$ (115/341) "strong" interaction to reconstruct 4) Curated_DBSCR(S). 5) Curated_RTB is the network from the RegTransBase database with 330 interactions, all of them labeled as "weak" since their experimental evidence was not available. Later, we merged Curated_FL, Curated_DBSCR, and Curated_RTB into 6) Curated_FL-DBSCR-RTB a meta-curated network comprising a total of 5386 genes and 9707 non-redundant regulatory interactions, which is the most extensive experimental GRN of $S$. coelicolor up to date. From this meta-curation, we filtered the "strong" interactions to reconstruct 7) Curated_FL(CS)-DBSCR(S). All curated networks are further described in Table 1. 


\section{The functional architecture of the S. coelicolor GRN}

To reveal the functional architecture and to elucidate the regulatory and biological function of some of the genes and interactions curated, we applied the Natural Decomposition Approach (NDA) on all the curated networks. The NDA is a biological-mathematical criterion to suggest a biological function of each gene based on the structure of the $\mathrm{GRN}^{20}$. It classifies the genes into one of the four structural classes: i) global regulators (GR), coordinating genes from different metabolic pathways ${ }^{25}$; ii) modular genes, group of genes working together to carry out a biological function ${ }^{20,26}$; iii) intermodular genes, integrating at the promoter level the response from different modules $^{20,27}$; and iv) genes constituting the basal machinery of the cell. We decided to further study the NDA analysis Curated_RTB-FL-DBSCR since it is the most complete GRN.

The NDA analysis of the meta-curated network Curated_RTB-FL-DBSCR revealed 20 GRs $(0.37 \%$ of the 5386 network genes), 502 modular genes (9.32\%), 18 intermodular genes (0.33\%), and 4846 basal machinery genes (89.97\%). The classification of each gene can be found at https://abasy.ccg.unam.mx/genes?regnetid=100226 v2019 sA22-DBSCR15-RTB13\&class=All.

Through the NDA analysis, we found 35 modules of genes. From them, module number 16 is a mega-module, which is divided into 12 submodules that are connected through the intermodular genes (Supplementary Figure 3a). To analyze the GRs identified in the meta-curated network, we reviewed the literature to identify the TFs that have been previously reported as global or pleiotropic regulators in S. coelicolor. Martín et al. ${ }^{28}$ reported a detailed description of the crosstalking between the global regulators in S. coelicolor and other Streptomyces. The review provides a list of genes considered as global and wide-domain regulators, due to the hundreds of genes they regulate and the multiple effects they produce ${ }^{28}$. Nine out of the 20 (45\%) GRs identified by the NDA were reported as such in this review. We further screened the literature to identify GRs or pleiotropic regulators reported in individual papers (Supplementary Table 4). We found 20 pleiotropic TFs or GRs reported individually, from which 13 (65\%) were categorized as GRs by the NDA. See the "Global regulators" section in Supplementary File 1 for further description of the GRs identified.

This analysis also revealed 18 intermodular genes. Some of their promoters integrate the signals of different GR related to carbon, nitrogen, and phosphate metabolism. For instance, glnA (SCO2198), gInII (SCO2210), and the amtB-gInK-gInD (SCO5583-85) operon, which are known to be mediators between the nitrogen and phosphate metabolism through the binding of their GR (PhoP and $G \operatorname{lnR}$ ) to these intermodular genes promoters ${ }^{29}$. Others integrate signals from primary and secondary metabolism, or morphological differentiation and antibiotic production. A further description of these genes can be found in Supplementary File 1 in the section "Intermodular genes". Moreover, the functional annotation of the modules identified by the NDA also provides a new functional hypothesis for genes whose function is currently unknown using a guilt-byassociation strategy (as previously described ${ }^{30}$ ). From the 46 modules and submodules in the GRN, $26 \%(12 / 46)$ are annotated. The annotation of each module can be found at https://abasy.ccg.unam.mx/modules?regnetid=100226 v2019 sA22-DBSCR15-RTB13. Most of the modules annotated are related to cellular metabolism, organic substances metabolism, and biosynthetic processes, which are fundamental processes for every cell (Supplementary Figure 2b). We found 245 with no previous annotation in $\mathrm{GOA}^{31}$ assigned to the annotated modules (Supplementary Table 5). 


\section{GRN inference based on binding sites identification performs better than that based on transcriptomics}

Despite the exhaustive curation, the meta-curated network Curated_FL-DBSCR-RTB has regulatory information for only $\sim 65 \%$ (5386/7825) of the S. coelicolor genome (network genomic coverage) and has $\sim 41 \%$ (9707/23908) of its expected total interactions (network interaction coverage or completeness) ${ }^{19}$, considering both "strong" and "weak" interactions. We leveraged the large corpus of high-throughput data available to computationally infer missing regulatory interactions to expand our GRN reconstruction. The inference was performed from two different approaches. For the first approach, we performed a regulon reconstruction, through the de novo prediction of TF binding sites and linked them to downstream genes. The regulon reconstruction was based on the network Curated_FL(CS)-DBSCR(S) using three methods for motif discovery: MEME, Bioprospector, and MDScan (see Methods). For the second approach, we performed a GRN inference from transcriptomic data. We used seven methods for GRN inference based on the gene expression data: CLR, Friedman, GENIE3, Inferelator, MRNET, Statmodel, and TIGRESS (see Methods and Supplementary Table 6a). These methods were selected based on their performance in a previous benchmarking of GRN inference methods ${ }^{18}$, their availability, complete documentation, and maintenance. For the inference, we selected an Affymetrix dataset (Platform GPL9417) from NCBI GEO ${ }^{33}$. See the "Transcriptomic Data" section in Supplementary File 1 for further description of the data selection. Since most of the experiments in the curation were performed on the S. coelicolor $\mathrm{A} 3(2)$ strain $\mathrm{M} 145$, which is plasmid-free, we restricted the inference to interactions among genes of the chromosome. Next, we evaluated the inferred GRNs computing the AUROC and the AUPR of the predictions (see Figure 3a and Supplementary Figure 4). From the AUPR, it is evident that in general GRN inference from binding sites performed better than the inference from expression data. For the inference from binding sites, MEME performed better than the other methods. For the inference from gene expression data, TIGRESS performed better, followed closely by GENIE3 and Inferelator. We assessed the inferred GRNs based mostly on the AUPR since it is more informative for imbalanced datasets ${ }^{36}$ as it is the case of GRNs inference $^{37}$ (Supplementary Figure 3a,b). Binding sites for each one of the interactions identified using MEME are reported in Supplementary File 3.

For the assessment, all the inferred networks were pruned to the 23908 best scoring interactions among genes part of the GS, since it is the number of interactions expected in the final network for S. coelicolor ${ }^{38}$. Nevertheless, the GS has only 387 interactions, which is $\sim 1.6 \%$ of the 23908 regulatory interactions expected in the complete regulatory network of S. coelicolor ${ }^{19}$. For this reason, the assessment only reflects the capacity of the methods to infer the interactions in the GS, while novel interactions (actual interactions not part of the incomplete GS) are labeled as false positives. Moreover, as the GS was used as prior for the regulon extension, it might provide an advantage for the network predicted by motif discovery. Because of its approach, inference by motif discovery predicts direct regulatory interactions, while inference from transcriptomic data predicts both direct and indirect ones without distinction. Thus, as the GS is only built by direct interactions, it is expected that inferred networks with the same type of interactions get a higher score. However, using the "non-strong" GRN as GS could drive to a bigger problem because indirect regulatory interactions might be spurious and are not adequate to assess causal interactions. 
Given that the current GS is still quite incomplete, and we cannot do proper discrimination among the different inferred networks, instead only using the single best method, we decided to build a community network for each one of the approaches. i) Inferred_BS for the prediction from binding sites; ii) Inferred_Exp for the prediction from expression data; iii) Inferred_BS-Exp, a community network from both previous community networks; and iv) Inferred_All, a community network built mixing individual networks from both approaches (see Table 1). For the latter, we used the three methods for binding site inference, along with Statmodel, GENIE3, and TIGRESS from expressionbased GRNs (due to their superior performance) to balance both approaches. Inferred_BS outperformed the rest of the community networks at both AUPR and AUROC (see Figure 3a). However, it was outperformed by MEME at both metrics. Given MEME's outstanding performance (see Figure 3a), we used it to perform a statistical validation of "weak" interactions supported by ChIP-data, similarly as proposed in ${ }^{22}$ (see Methods). A total of 55 "weak" interactions were reclassified as "strong" (see Figure $3 \mathrm{~b}$ and Supplementary Table 6b). We found one of these interactions (SCO4230-SCO4878) already reported as "strong" in the DBSCR database (Curated_DBSCR(S)). These statistically validated interactions were merged with the "strong" interactions from Curated_FL and from the meta-curated network Curated_FL-DBSCR-RTB into two networks: Curated_FL(S) and Curated_FL(S)-DBSCR(S). We reassessed the network predictions with Curated_FL(S)-DBSCR(S) as GS and the results remained virtually the same (Supplementary Figure 5). For completeness, we also performed the assessment using Curated_FL-DBSCR15-RTB13 as the GS (Supplementary Figure 6). However, the 72 regulators in Curated_FL(S)-DBSCR(S) are the only TFs that were used for the predictions based on binding sites. On the other hand, Curated_FLDBSCR15-RTB13 has 137 TF. This resulted in a poor recall by the predictions based on binding sites (Supplementary Figure 6), as interactions for 65 TFs are not predictable because their regulations might be carried out indirectly, with no need for a DNA binding site.

\section{Inferred networks have a similar structure to the largest curated networks}

Even though the AUPR and AUROC metrics allow the assessment of the predictions, both metrics heavily rely on the ranking of the predicted interactions. Moreover, the GS is not complete and missing interactions would be still classified as false positives, decreasing the score more as higher its ranking is. We also assessed the inferences in terms of their structural properties and compared them against the curated networks to compensate for such drawbacks. Note that this approach has its caveats. The global structural properties of the network might be different once the GS is complete, this can be approached by comparing the predictions to all the curated networks, each of them with different completeness. Also, two networks could have the same topology with different node entities. For this reason, we use the topological assessment in complement with the AUPR and AUROC metrics to identify the best prediction.

One of the main characteristics of biological networks is that they are scale-free and hierarchically modular. Same characteristics that our curated networks have been proved to possess (Supplementary File 1). Therefore, as an initial approach, we asked whether the inferred networks are scale-free too. These are networks whose degree (nodes' connectivity) distribution $P(k)$ follows a power law, $P(k) \sim k^{-\alpha}$, with $2<\alpha<3^{20,38,39}$. This since with an $\alpha=2$ appears a unique global regulator (hub-and-spoke network) and for $\alpha>3$ scale-free networks lose most of their characteristic properties ${ }^{39}$. First, to compute this $\alpha$ for the inferred networks, we performed a robust linear regression over a log-log plot of the complementary cumulative degree distribution 
and corrected the exponent accordingly (see Table 2 and Supplementary Figures 7-11). All inferred networks' degree distribution seems to follow a power law according to the adjusted coefficient of determination. Nevertheless, the data points in Inferred_All appear to be divided into three regions with different tendencies, instead of the two that are present in the other networks (Supplementary Figures 7-11). Usually, this type of network is divided into two regions, the region of the nodes (genes) with a low degree, and the one with nodes with a high degree ${ }^{40}$. The appearance of a third region might be a consequence of merging networks from methods with different approaches. This could affect the structural properties of the merged networks, while communities from the same approach appear to have more similar structural properties. In the case of Inferred_BS-Exp, the construction of communities ahead by each approach create more compatible networks in terms of structure that can be conveniently mixed.

Next, we wanted to confirm that their degree distribution followed a power law, so we contrasted each potential power law of the inferred networks the distributions against alternative fat-tailed probability distributions (Power-law, exponential, stretched exponential, lognormal, and truncated power-law) using Kolmogorov-Smirnov tests ${ }^{38,41}$ to confirm that the degree distributions follow a power law (Supplementary Table 6a). We found the degree distribution of the inferred networks adjusted better to a power-law distribution than to an alternative distribution. Then, we computed a maximum likelihood estimation for the exponent $(\alpha)$ of the power law and found that most of them are between two and three, except for Inferred_All showing it as an anomalous scale-free network (Supplementary Table 7). Perhaps caused by the mixing of networks with diverse structural properties. Nevertheless, we could consider all inferred networks to be scalefree.

However, we wanted to check the other properties of scale-free networks (see Table 2 ) $^{39,42}$. The four community networks have small average shortest path lengths and a high clustering coefficient. Inferred_BS has the smallest average short path length, while Inferred_All has the highest average clustering coefficient (see Table 2). Scale-free networks also present an ultra-small world effect, which implies that the average path length is proportional to $\ln (\ln (N))$ ( $\mathrm{N}$ is the number of nodes in the network). This is the case for all the inferred networks. Another characteristic of GRNs is their hierarchical modularity, which implies a diamond-shaped hierarchical organization as has been revealed by the $\mathrm{NDA}^{20}$. In a scale-free network, this implies that the clustering coefficient depending on the degree $(C)$ follows a power law as $C(k) \sim k^{-139}$. Inferred_BS has the exponent closest to -1 (0.92), with the best $R^{2}$. Even though Inferred_BS seems to be the network that behaves closest to a GRN, all networks have similar values, which makes it difficult to discern the most reliable inferred network.

To perform a more thorough comparison of their structural graph properties, we include several other (Supplementary Table 8). We clustered their vectors of structural properties for the curated and community-inferred networks (see Figure 4a). The clustering partitions the networks into two major groups. The first one contains the curated networks and Inferred_BS, while the second group contains the other inferred networks. The first group is in turn also divided into two groups: one with the two largest curated networks and Inferred_BS, and the other one with the remaining curated networks. The reason for this may be due to the size of the networks (see Table 1). When standardizing the property values by max-min feature scaling, the two largest curated networks were clustered with the predictions (Supplementary Figure 12), which could be also due to the size 
of the network. To reduce the network size influence we used the network dissimilarity measure proposed by Schieber et al. ${ }^{43}$. We considered the third term which makes the distance measure robust to graph size in terms of the number of nodes (genes) ${ }^{43}$ (see Figure $4 \mathrm{~b}$ ). Even with this metric, the two largest curated networks were clustered with the inferred networks. This might be a consequence of the high fraction of maximum out-connectivity and structural genes in the largest curated networks, like those found in the inferred networks. This shows that the inferred networks are similar that the most complete curated networks in terms of structure, suggesting their reliability.

\section{The Natural Decomposition Approach identifies regulatory networks similarity despite their different completeness level}

We compared all curated and community inferred networks based on the Simpson similarity of the four components proposed by the NDA: global regulators, modular genes, intermodular genes, and the basal machinery (see Figure 5). The Simpson similarity index measures the size of the core of two sets with reference to the smallest one ${ }^{44}$. Note that two identical sets, being one a subset of the other one will have a score of 1 . On the other hand, two completely different sets will have a score of 0 .

When comparing the global regulators (see Figure $5 a$ ), there is not a distinct division among the networks. Infered_BS-Exp and Infered_Exp have a similar correlation with all the curated networks, slightly higher with Curated_DBSCR(S), Curated_FL, and Curated_FL-DBSCR-RTB. These two inferred networks have the highest amount of GR, 116, and 114 respectively; thus, the other GRs predicted could be easily a subset of them. For the case of Infered_BS, it has the highest correlation with the "strong" networks. This is expected since these networks have only direct regulatory interactions, as the interactions predicted in Inferred_BS, while there is no evidence of direct regulation for transcriptomic-based inferred interactions. This can affect the measurement of the effect of GRs over the rest of the genes since GRs regulating not so many targets from different processes might not be predicted as such in the "strong" networks. However, when their indirect influence is represented in the network, their ranking as GRs is noticeable.

When analyzing the modular genes, there are two major groups (see Figure $5 b$ ): the major group, with the curated networks, is divided into two subgroups, one with Curated_RTB, Curated_DBSCR, and its "strong" version Curated_DBSCR(S), and the second subgroup contains the integration proposed in this work. Interestingly, the meta-curated network Curated_FL-DBSCR-RTB correlates very well with all the smaller networks it contains, from which we could deduce that modular genes are conserved despite the addition of new regulatory interactions. In the second group, composed of the inferred networks, we can see there is not a high correlation among them. Inferred_BS is the closest to the curated networks, while Inferred_Exp and Inferred_BS-Exp have a high correlation. This tells us that the interactions in Inferred_Exp have a larger influence on the module configuration of Inferred_BS-Exp than Inferred_BS. The difference between the curated and inferred networks might come from the fact that inferred networks have a greater number of GRs and a much lower number of modular genes when compared with the curated networks (Supplementary Table 9).

Intermodular genes are the less conserved NDA class (see Figure 5c). There is overlap only among the smallest curated networks, all share the intermodular de gene SCO5877, which appears as a TF 
in the other curated networks. Moreover, an overlap among Curated_FL and Curated_FL-DBSCR$R T B$, which share most of the interactions. Thus, is expected that they also share most of the intermodular genes. Note that the networks Curated_DBSCR(S) and Inferred_BS-Exp are not included in the clustering since they did not present any intermodular genes. Finally, when analyzing the basal machinery (see Figure $5 \mathrm{~d}$ ), the larger curated networks are grouped on one side, next to the inferred networks, and finally the smallest curated networks with Curated_RTB as an outgroup. Even though Inferred_BS is grouped with the other inferred networks, it has a higher correlation with Curated_FL(S) and Curated_FL(S)-DBSCR(S), which again evidence the similarity among these three networks.

\section{Assessment of the global regulators' inference}

In the NDA, the identification of global regulators is a key step in the classification of every node in the GRN. Previously, it has been reported a high overlap between the predictions of global regulators by the NDA and those reported in the literature for E. coli ${ }^{20}$, B. subtilis ${ }^{45}$, and C. glutamicum ${ }^{27}$. We used the set of GRs reported by Martín et. Al. ${ }^{28}$, besides those reported in independent articles, and the union of both sets (Supplementary Table 4). Then we assessed the predictions of the GR using the Matthews correlation coefficient (MCC) (see Figure 6) We used the MCC score as it is more informative and reliable than F1 for binary classification evaluation ${ }^{46}$, however when applying the F1 score we obtained consistent results (Supplementary Figure 13).

Curated_FL and Curated_RTB-FL-DBSCR have the best performance in GR prediction. However, the "strong" networks have a slightly smaller score even having much less genomic coverage. This shows that the GRs are very robust to perturbations in the network as previously shown ${ }^{20}$. On the other hand, despite the high coverage of the inferred networks, the performance of the predictions with such networks was poor. This could be, as it was mentioned before, due to the great amount of GR predicted by these networks, which would cause a high proportion of false positives, affecting the score. Inferred_BS produced the most conservative prediction (lowest false-positives rate) among the inferred network (Supplementary Figure 14 and Supplementary Table 9).

\section{GRN inference from transcription factor binding sites proves to be the most reliable approach and allows the prediction of new TFs for the most studied SARPs}

Even though other similar studies suggest the integration of inference approaches as the most suitable methodology for GRN reconstruction ${ }^{18,47,48}$, because of the analysis performed in this paper, we consider Inferred_BS as the most reliable inferred network. From the evaluation against the GS, where it presented the highest AUPR and AUROC among the community networks, through its structural properties along with the NDA analysis where it showed the most similar configuration to a GRN reconstruct from biological experiments. Moreover, it has the largest genomic coverage among all the networks, which would be advantageous for a deeper study of transcriptional regulation in S. coelicolor. Therefore, we decided to use inferred_BS to further study the regulation of the SARPs of the most studied antibiotics in S. coelicolor: Actll-orf4, RedD/RedZ, CpkO (also known as KasO), and CdaR, which regulate the production of ACT, RED, $y C P K$, and CAD, respectively ${ }^{3}$ (Supplementary Table 10). A total of 13 new putative regulators for the SARPs were predicted, providing us a great opportunity to find new targets to manipulate the 
S. coelicolor antibiotic production. As it is not possible to support computationally a direct binding of this regulator, it is necessary to corroborate them with wet-lab experiments.

Following, we describe some of the TFs predicted for the SARPs using the workflow suggested in Figure 1b: For actII-orf4 (SCO5085) only one novel regulator was inferred, MacR (SCO2120) which is the response regulator of the TCS MacRS, while the rest was already part of Curated_FS(S)$D B S C R(S)$ (Supplementary Figure 15). This TCS has been proved to activate ACT production. Nevertheless, a ChIP-qPCR analysis was not able to prove an in vivo interaction between MacR and actII-orf4, although a direct binding was not tested as Inferred_BS predicted ${ }^{49}$. For redD (SCO5877) two new regulators were predicted, LipR (SC00712) and Actll-orf4 (SCO5085). LipR is related to AfsR $(S C 04426)^{50}$, homolog to the SARPs, and activator of the ACT and RED production ${ }^{51}$. Moreover, its mutant affects ACT production ${ }^{50}$, which makes it plausible to affect RED production as well. It has been suggested that ActIl-orf4 might regulate the production of other antibiotics ${ }^{4}$, which could be by binding directly to their CSR. For redZ (SCO5881) five new regulators were predicted, among them is GluR (SCO5778) which has been shown to affect RED production. Nevertheless, it has been shown that GluR does not bind directly to redZ, thus it could be more an indirect regulation ${ }^{52}$. Another one, StgR (SCO2964) has been shown, by an RT-qPCR experiment, to be a repressor of redD ${ }^{53}$, thus this repression could be through the direct binding to redZ. $\mathrm{HpdA}$ (SCO2928) and HpdR (SCO2935) are related to Tyrosine catabolism, which produces important precursors for antibiotic biosynthesis ${ }^{54}$. Moreover, HpdA has been shown to activate actII-ORF4, therefore might have a more direct role in RED production. In the case of cdaR (SCO3217), we have four predicted regulators, among them, OsdR (SC00204) and RamR (SC06685). Both are related to the response to stress and the development of S. coelicolor ${ }^{55,56}$. SsgR (SCO3925) regulates the sporulation and morphological differentiation ${ }^{57}$. These all processes are highly related to antibiotic production. Finally, for cpkO/kasO (SC06280) six new regulators were inferred, among them OsdR (SCO0204), LipR (SC00712), and StgR (SCO2964) were described before. Another one is NnaR (SCO2958), which regulates spore formation and antibiotic production $^{58}$. We refer the reader to the Supplementary material for the complete list of the predicted regulators in every predicted network (Supplementary Table 10). We further compared the six regulators for actll-orf4 identified by Inferred_BS with the 11 TFs found in Inferred_All, from which only two were part of Curated_FS(S)-DBSCR(S) and three more were included in Curated_FS-DBSCR-RTB (Supplementary Figure 15,16). We found that the interactions included in Inferred_All tend to be carried out indirectly (i.e., not through a TF-DNA interaction), suggested by a poor overlap between Inferred_BS and Inferred_All, and biological insights such as the putative regulation of actll-orf4 by OsdR.

\section{Comparative analysis with Corynebacterium glutamicum shows coherent system-level components conservation}

The diamond-shaped structure identified by the NDA is conserved between E. coli and B. subtilis ${ }^{45}$. As an application of the meta-curated network, we studied the conservation of its system-level components, comparing it against the $C$. glutamicum network, which is phylogenetically related to S. coelicolor, and a model organism for the study of $\mathrm{GRNs}^{27}$. We applied the regulogs analysis ${ }^{59}$ with one-to-one orthology relationships to alleviate network incompleteness and make them 
comparable. As prior networks, we used Curated_FL(S)-DBSCR(S) (534 interactions) for S. coelicolor and 196627_v2020_s21_eStrong from Abasy Atlas ${ }^{19}$ (2941 interactions) for C. glutamicum ${ }^{16}$. After the regulogs analysis, we ended up with 2966 interactions in C. glutamicum and 692 interactions in S. coelicolor.

We used the complemented networks to identify GRN-wide orthologous relationships defined as the orthologous present in the GRN of the respective organism. We obtained a total of $188 \mathrm{GRN}-$ wide orthologous relationships from a total of 995 1:1 orthologs identified by OrthoFinder ${ }^{60}$. We applied the NDA analysis to both GRNs to identify the system-level components and computed the fraction of the GRN-wide orthologous in each combinatory relationship between the components (see Figure 7a). We found that most of the GRN-wide orthologous (54\%) are classified as basal machinery in both organisms. This is expected since $73 \%$ and $74 \%$ of the genes correspond to the basal machinery in the complemented networks of $C$. glutamicum and S. coelicolor, respectively. Besides, the distribution of the genes in the chromosome of $S$. coelicolor shows a central core, where are genes likely related to primary functions such as DNA replication, transcription, translation, and amino-acid biosynthesis; and likely non-essential genes such as secondary metabolism are in the chromosome arms ${ }^{5}$. More than 59\% (111/188) of the GRN-wide orthologs conserved the same class in both organisms (Figure 7a) showing high conservation of the NDA classification.

We studied the pairwise Simpson similarity index between the four classes between the two organisms to remove the problem of the imbalanced classes in both organisms (see Figure 7b). GR is the class with the highest conservation rate, the orthologs of seven of the eight GRs in C. glutamicum are also GRs in S. coelicolor (see Figure 7b and Figure 7c). The conservation between the same class in the two organisms is also high for the basal machinery, while poor for the modular genes. For the case of intermodular genes, even though the networks were complemented with information from the other network, they are not conserved at all (see Figure 7b). Previous work reported intermodular genes as the least conserved of the system-level components $^{27}$. Intermodular genes are the most likely responsible for giving the TRN flexibility and increasing evolvability by scouting different combinations of regulatory interactions between physiological functions so the organism could adapt better to environmental changes ${ }^{45}$. These results agree with a previous analysis of the robustness of the NDA to a random node and edge remotion showing GR and intermodular genes as the most and least conserved classes, respectively ${ }^{27}$.

On the other hand, $24 \%$ of the GRN-wide orthologs that are modular genes in C. glutamicum were classified in S. coelicolor as basal machinery. This could be due to three possible reasons ${ }^{45}:$ i) the basal machinery genes in $S$. coelicolor are misclassified and further research is needed to find the missing regulatory interactions (see Figure 7c) that will integrate some of the basal machinery genes into a module. ii) The GRs controlling C. glutamicum genes are not yet identified as GRs. iii) Genes in S. coelicolor need a more direct regulation because of their physiological function (high plasticity of transcriptional regulation). The previous comparison between S.coelicolor, Mycobacterium tuberculosis, and Corynebacterium diphtheriae showed a synteny among the whole chromosome of these last two microorganisms and the core of the one of S. coelicolor. C. glutamicum is phylogenetically closely related to_M.tuberculosis and $C$. diphtheriae, with roughly similar genome size. Therefore, a similar result would be expected. Furthermore, as more 
classical experiment data become available, new regulations for the currently basal machinery would turn those genes into the modular class. However, a deeper analysis of diverse factors such as genome size, the niche of the organisms, and a wider range of organisms are required to further study the robustness of the NDA analysis.

\section{Conclusions}

A meta-curated regulatory network for S. coelicolor (Curated_RTB-FL-DBSCR) was reconstructed from a collection and curation of regulatory interactions experiments in literature and databases. From the NDA analysis of the meta-curated network, we could identify 20 global regulators, of which $95 \%$ (19/20) have already been reported as global or pleiotropic regulators. 46 functional modules were identified and 18 intermodular genes, some of them found to be involved in more than one biological process. Functional modules annotated by GO enrichment allowed via a 'guilt by association' strategy to propose a function for 245 genes without any previous functional annotation or annotated as 'hypothetical protein'. This network is, however $\sim 42 \%$ of the estimated complete regulatory network, which evidences a lack of information related to S. coelicolor transcriptional regulation. Especially for interactions experimentally supported by "strong" evidence, which accounts for only $\sim 2 \%$ of the estimated complete network. We indeed found a low level of direct experimental validation for the regulatory interactions reported in the literature and curated in this work as only 6\% (533/9687) are supported by experiments confirming the binding of the TF to the upstream region of the target gene, the so-called "strong" evidence. This highlights the importance of carrying on more experiments aimed to confirm the weakly supported interactions to increase our knowledge of the transcriptional regulation in $S$. coelicolor. Notwithstanding, the meta-curated network Curated_RTB-FL-DBSCR provides the most extensive and up-to-date reconstruction available for the regulatory circuitry in this organism and already portrays accurately the functional organization of $S$. coelicolor regulation. GRN inference from transcriptomic and DNA sequence data was performed and the inference from TF binding sites identification showed to be the best approach according to interactions inference assessment, topological assessment, and systems-level comparison. This final inferred network is a valuable guide for wet-lab experiments, since narrows down the search space of the possible TF for each gene. Besides, it can be used in computational models of $S$. coelicolor. From this network 13 new TFs were predicted to bind in the upstream region of five of the principal SARPs, most of which previously proved to affect indirectly antibiotic production or be related to stress response or morphological differentiation. Finally, we compared S. coelicolor network to C. glutamicum GRN, showing one of the many potential applications of the curated network. There we found high conservation only for the basal machinery, which might be a result of the high plasticity of the transcriptional regulation. We strongly suggest using Abasy Atlas to visually explore the interactions validated by experiments and identify the role of the genes in the global regulatory network (e.g., global/local regulators) and functional annotation.

\section{Material and methods}

\section{Transcriptional regulatory interactions curation}

We performed a comprehensive review of the literature to identify experimentally-supported transcriptional regulatory interactions in Streptomyces coelicolor A3(2). We searched peerreviewed articles in Google Scholar and PubMed using the keywords "Streptomyces coelicolor" 
AND "transcriptional" and its variations AND "regulation" and its variations. In the case where reviews were found, their references were followed to the original research papers. Then, we performed the curation and organized the interactions (Supplementary File 1). Experiments were classified according to their methodology and their names were standardized for the sake of clarity and easier evidence classification. We merged these interactions with two previously curated networks, one was reconstructed from an XML provided by the DBSCR team and the other one from RegTransBase ${ }^{24}$ available at the Abasy Atlas website. These datasets are available from http://dbscr.hgc.jp/ and https://abasy.ccg.unam.mx. Abasy Atlas is a database of meta-curated bacterial GRNs for nine species including $S$. coelicolor ${ }^{19}$. It also provides historical snapshots for other model organisms such as Escherichia coli, Bacillus subtilis, Corynebacterium glutamicum, and Mycobacterium tuberculosis ${ }^{19}$.

\section{GRN inference from transcription factor binding sites}

To extend the regulons for the TFs identified in the literature, we used the set of "strong" interactions as prior (Curated_FL(CS)-DBSCR(S)). We reconstructed a position weight matrix (PWM) for every TF in the "strong" network using the non-overlapping up to -300 to +50 bp (with reference to the translation start codon) upstream regions of their TGs as input for three motif discovery algorithms. Namely, i) MEME, an extension of the expectation-maximization algorithm for fitting finite mixture models ${ }^{61}$; ii) BioProspector, based on multiple Gibbs sampling ${ }^{62}$; and iii) MDscan, that employs a heuristic word-enumeration approach combined with statistical modeling $^{63}$. Then, we used FIMO ${ }^{64}$ ( $p$-value threshold $=1 \times 10^{-4}$ ) to identify TF-TG interactions. As most of the interactions curated are from S. coelicolor A3(2) strain M145 (plasmid-free), we excluded genes that are not part of the chromosome.

\section{GRN inference from transcriptomic data}

We downloaded first the transcriptomic dataset for S. coelicolor available at the COLOMBOS database ${ }^{32}$. Then, we also download data from the NCBI Gene Expression Omnibus (GEO) ${ }^{33}$. From there we download an Affymetrix dataset (Platform GPL9417) and an RNA-Seq dataset (GPL26763. Afterward, we normalize the Affymetrix data using Robust Multi-chip Averaging (RMA) with the affy package ${ }^{65}$ and used the gPCA package ${ }^{66}$ to identify a batch effect in the data, which was corrected with Combat from the sva package ${ }^{67}$, all of them are packages for $R$. The data counts on 137 transcriptomes for 7738 genes. As in the case of GRN inference from transcription factor binding sites, we only considered genes from the chromosome. We selected the best inference methods according to their outstanding performance in the DREAM challenge ${ }^{18}$. Moreover, we selected methods that have an implementation in $\mathrm{R}$ or Matlab and were well documented. The inference methods selected were: i) $\mathrm{CLR}^{68}$, a method that applies mutual information; ii) GENIE ${ }^{69}$, which applies tree-based regression and feature selection; iii) Inferelator ${ }^{70}$, which applies regression and variable selection; iv) $\mathrm{MRNET}^{71}$, which applies the maximum relevance/minimum redundancy algorithm; and v) TIGRESS ${ }^{72}$ which applies LARS combined with stability selection. Along with these methods, we used two modifications we propose in this work, Friedman, and Statmodel (Supplementary File 1). We provided all methods with a list of 137 TFs from the metacurated network Curated_FL-DBSCR-RTB to infer causality ${ }^{18,72}$. 


\section{Integration of individual inferences into a community GRN}

To increase the precision of the predictions we used a community approach ${ }^{18}$ integrating individual predictions from different algorithms. First, the individual predictions are sorted by their confidence score, keeping the most reliable ones at the beginning of the prediction list. Then, the average of the rank positions in the predictions is given as the community score for each interaction. For missing interactions in a prediction list, the position is equal to the size of the prediction list +1 . All community networks were pruned to the 23908 first interactions with the highest score, which is the predicted size of the complete GRN of $S$. coelicolor reported by Abasy Atlas $\mathrm{v} 2.4^{19}$ according to the model developed $\mathrm{in}^{38}$. The model is constantly being updated on the Abasy Atlas website by the addition of new networks and interactions, which will cause a slight variation in the number of interactions ${ }^{19}$.

\section{Assessment of the inferred GRN}

We computed the area under the precision-recall curve (AUPR) and the area under the receiver operating characteristics curve (AUROC) to assess our predictions using in-house scripts. The AUPR depicts the precision (1) as a function of the recall (2) obtained by the predictor. The AUROC depicts the relation between the recall, also called the true positive rate (TPR) (2), and the false positive rate (FPR) (3). Note that unknown actual interactions between genes in the GS will still be considered as $\mathrm{FP}^{37}$. For this reason, interactions involving genes that are not part of the GS were not considered.

$$
\begin{gathered}
\text { Precision }=\frac{T P}{T P+F P} \\
\text { Recall }=T P R=\frac{T P}{T P+F N} \\
F P R=\frac{F P}{F P+T N}
\end{gathered}
$$

\section{Statistical validation for interactions supported by ChIP experiments.}

We performed a statistical validation approach for ChIP-data ${ }^{22}$ for those interactions supported by ChIP experiments and no "strong" experiment. Because of the lack of data in some articles, instead of performing the statistical validation finding the motifs from the ChIP data directly as $\mathrm{in}^{22}$, we used the "strong" interactions as seed to construct the matrix models for the TFs. We used $M E M E^{61}$ to build a position weight matrix (PWM) for each TF with at least 3 "strong" interactions. Then, we used FIMO with the matrix for each TF to scan the non-overlapping up to -300 to +50 bp upstream regions with reference to the translation start sites of S. coelicolor. We kept those TF binding sites with $\mathrm{p}$-value $<1 \times 10^{-4}$ and used these interactions to compare them with those supported by ChIP technologies, keeping the intersection of both sets (interactions supported by both ChIP and motif finding approaches). 


\section{Network similarity}

We computed the characteristic structural properties for GRNs reported as global properties on the Abasy Atlas database ${ }^{19}$. Namely, regulators $\left(k_{\text {out }}>0\right)(\%)$, direct regulatory interactions, selfregulation (\%), maximum out-connectivity (\%), network density, weakly connected components, genes in the giant component (\%), feedforward circuits, complex feedforward circuits, 3-Feedback loops, average shortest path length, network diameter, average clustering coefficient, adjusted coefficient of determination $\left(R_{a d j}^{2}\right)$ of $P(k)$, and $R_{a d j}^{2}$ of $C(k)$. Then we used pairwise Pearson correlation among the profiles of the structural properties of the networks and cluster them according to the Euclidean distance among the correlations using Ward's method. To compute the pairwise network dissimilarity we used a Python implementation adapted from that proposed by Schieber et al. ${ }^{43}$ with the parameters proposed by the authors $(0.45,0.45,0.1)$, being the last one required to discriminate network size in terms of nodes (genes) ${ }^{43}$. Then, we clustered the networks by using Euclidean distances among its dissimilarity values using Ward's variance minimization algorithm as the linkage method.

\section{System-level components}

We applied the Natural Decomposition Approach (NDA), a biological-mathematical criterion to identify the components of the diamond-shaped structure of the GRNs ${ }^{20}$, on every curated and inferred network. See the Supplementary methods (Supplementary File 1) for a brief description of the NDA, and ${ }^{20}$ for further details. The assessment of the prediction of GRs was performed using in-house scripts for MCC, precision, and F1-score. Scores were computed for each GRs prediction obtained by the NDA with the different networks analyzed in this work. As GS we used the GRs previously reported in the literature from a review ${ }^{28}$, from individual publications, and both (Supplementary Table 4).

\section{Comparative analysis against $C$. glutamicum}

We used as prior the strong regulatory networks Curated_FL(S)-DBSCR(S) for S. coelicolor, and 196627_v2020_s21_eStrong from the Abasy Atlas database for C. glutamicum ${ }^{19}$, considering only the interactions between two genes both mapping to a locus tag. We used MEME to construct a PWM for every TF with at least three TGs using their upstream sequences. These sequences were defined as the non-overlapping regions of up to -300 to $+50 \mathrm{bp}$ with reference to the translation start codon and were obtained with retrieve-seq from $\mathrm{RSAT}^{73}$. Then, we used FIMO with the PWM of the TFs from S. coelicolor to find individual occurrences with a $\mathrm{p}$-value $<1 \times 10^{-4}$ in the upstream sequences of $C$. glutamicum. The same was done in the opposite direction. With this, we seek to alleviate network incompleteness by extrapolating known interactions from an organism to the other $^{59}$. Predicted interactions were sorted by $\mathrm{p}$-value and only the best scoring result was conserved for redundant interactions. Afterward, we used Orthofinder to find one-to-one ortholog relationships between both organisms. We used OrthoFinder due to its high accuracy ${ }^{60}$. The orthologs were used to further filter FIMO predictions to conserve interactions in which both TF and TG have a one-to-one orthologous relationship in the other organism. We considered the original "strong" network interactions at the beginning of the interactions list. The NDA was applied to both expanded GRNs to identify ortholog systems and only the genes with one-to-one orthologs in the other organism's network (GRN-wide orthologs) were considered in the analysis. 


\section{Availability of supporting data}

The data set(s) supporting the results of this article are included within the article, its additional Files, or in Abasy Atlas at https://abasy.ccg.unam.mx/.

\section{References}

1 Hoskisson, P. A. \& van Wezel, G. P. Streptomyces coelicolor. Trends Microbiol 27, 468-469, doi:10.1016/j.tim.2018.12.008 (2019).

2 Mast, Y. \& Stegmann, E. Actinomycetes: The Antibiotics Producers. Antibiotics (Basel) 8, doi:10.3390/antibiotics8030105 (2019).

3 Chen, S. et al. Roles of two-component system AfsQ1/Q2 in regulating biosynthesis of the yellow-pigmented coelimycin P2 in Streptomyces coelicolor. FEMS Microbiol Lett 363, doi:10.1093/femsle/fnw160 (2016).

4 McLean, T. C., Wilkinson, B., Hutchings, M. I. \& Devine, R. Dissolution of the Disparate: Coordinate Regulation in Antibiotic Biosynthesis. Antibiotics (Basel) 8, doi:10.3390/antibiotics8020083 (2019).

5 Bentley, S. D. et al. Complete genome sequence of the model actinomycete Streptomyces coelicolor A3(2). Nature 417, 141-147, doi:10.1038/417141a (2002).

6 Bednarz, B., Kotowska, M. \& Pawlik, K. J. Multi-level regulation of coelimycin synthesis in Streptomyces coelicolor A3(2). Appl Microbiol Biotechnol 103, 6423-6434, doi:10.1007/s00253-019-09975-w (2019).

7 Bibb, M. 1995 Colworth Prize Lecture. The regulation of antibiotic production in Streptomyces coelicolor A3(2). Microbiology 142 ( Pt 6), 1335-1344, doi:10.1099/13500872-142-6-1335 (1996).

8 Chater, K. F. Regulation of sporulation in Streptomyces coelicolor A3(2): a checkpoint multiplex? Curr Opin Microbiol 4, 667-673, doi:10.1016/s1369-5274(01)00267-3 (2001).

9 Bibb, M. J. Regulation of secondary metabolism in streptomycetes. Curr Opin Microbiol 8, 208-215, doi:10.1016/j.mib.2005.02.016 (2005).

10 Martin, J. F. \& Liras, P. Cascades and networks of regulatory genes that control antibiotic biosynthesis. Subcell Biochem 64, 115-138, doi:10.1007/978-94-007-5055-5_6 (2012).

11 Zitnik, S., Zitnik, M., Zupan, B. \& Bajec, M. Sieve-based relation extraction of gene regulatory networks from biological literature. BMC Bioinformatics 16 Suppl 16, S1, doi:10.1186/1471-2105-16-S16-S1 (2015).

12 Novichkov, P. S. et al. RegPrecise 3.0--a resource for genome-scale exploration of transcriptional regulation in bacteria. BMC Genomics 14, 745, doi:10.1186/1471-2164-14745 (2013).

13 Leyn, S. A. et al. Comparative genomics and evolution of transcriptional regulons in Proteobacteria. Microb Genom 2, e000061, doi:10.1099/mgen.0.000061 (2016).

14 Metris, A. et al. SalmoNet, an integrated network of ten Salmonella enterica strains reveals common and distinct pathways to host adaptation. NPJ Syst Biol Appl 3, 31, doi:10.1038/s41540-017-0034-z (2017).

15 Staunton, P. M., Miranda-CasoLuengo, A. A., Loftus, B. J. \& Gormley, I. C. BINDER: computationally inferring a gene regulatory network for Mycobacterium abscessus. $B M C$ Bioinformatics 20, 466, doi:10.1186/s12859-019-3042-8 (2019).

16 Escorcia-Rodríguez, J. M., Tauch, A. \& Freyre-González, J. A. <em>Corynebacterium glutamicum $</ e m>$ regulation beyond transcription: Organizing principles and 
reconstruction of an extended regulatory network incorporating regulations mediated by small RNA and protein-protein interactions. bioRxiv, 2021.2001.2007.423633, doi:10.1101/2021.01.07.423633 (2021).

17 Castro-Melchor, M., Charaniya, S., Karypis, G., Takano, E. \& Hu, W. S. Genome-wide inference of regulatory networks in Streptomyces coelicolor. BMC Genomics 11, 578, doi:10.1186/1471-2164-11-578 (2010).

18 Marbach, D. et al. Wisdom of crowds for robust gene network inference. Nat Methods $\mathbf{9}$, 796-804, doi:10.1038/nmeth.2016 (2012).

19 Escorcia-Rodriguez, J. M., Tauch, A. \& Freyre-Gonzalez, J. A. Abasy Atlas v2.2: The most comprehensive and up-to-date inventory of meta-curated, historical, bacterial regulatory networks, their completeness and system-level characterization. Comput Struct Biotechnol J 18, 1228-1237, doi:10.1016/j.csbj.2020.05.015 (2020).

20 Freyre-Gonzalez, J. A., Alonso-Pavon, J. A., Trevino-Quintanilla, L. G. \& Collado-Vides, J. Functional architecture of Escherichia coli: new insights provided by a natural decomposition approach. Genome Biol 9, R154, doi:10.1186/gb-2008-9-10-r154 (2008).

21 Santos-Zavaleta, A. et al. RegulonDB $\vee$ 10.5: tackling challenges to unify classic and high throughput knowledge of gene regulation in E. coli K-12. Nucleic Acids Res 47, D212-D220, doi:10.1093/nar/gky1077 (2019).

22 Weiss, V. et al. Evidence classification of high-throughput protocols and confidence integration in RegulonDB. Database (Oxford) 2013, bas059, doi:10.1093/database/bas059 (2013).

23 Park, S. S. et al. Mass spectrometric screening of transcriptional regulators involved in antibiotic biosynthesis in Streptomyces coelicolor A3(2). J Ind Microbiol Biotechnol 36, 1073-1083, doi:10.1007/s10295-009-0591-2 (2009).

24 Cipriano, M. J. et al. RegTransBase--a database of regulatory sequences and interactions based on literature: a resource for investigating transcriptional regulation in prokaryotes. BMC Genomics 14, 213, doi:10.1186/1471-2164-14-213 (2013).

25 Gottesman, S. Bacterial regulation: global regulatory networks. Annu Rev Genet 18, 415441, doi:10.1146/annurev.ge.18.120184.002215 (1984).

26 Hartwell, L. H., Hopfield, J. J., Leibler, S. \& Murray, A. W. From molecular to modular cell biology. Nature 402, C47-52, doi:10.1038/35011540 (1999).

27 Freyre-Gonzalez, J. A. \& Tauch, A. Functional architecture and global properties of the Corynebacterium glutamicum regulatory network: Novel insights from a dataset with a high genomic coverage. J Biotechnol 257, 199-210, doi:10.1016/j.jbiotec.2016.10.025 (2017).

28 Martín, J. F., Santos-Beneit, F., Sola-Landa, A. \& Liras, P. in Stress and Environmental Regulation of Gene Expression and Adaptation in Bacteria (ed Frans J. de Bruijn) 257-267 (John Wiley \& Sons, Inc., 2016).

29 Martin, J. F. et al. Cross-talk of global nutritional regulators in the control of primary and secondary metabolism in Streptomyces. Microb Biotechnol 4, 165-174, doi:10.1111/j.1751-7915.2010.00235.x (2011).

30 Ibarra-Arellano, M. A., Campos-Gonzalez, A. I., Trevino-Quintanilla, L. G., Tauch, A. \& Freyre-Gonzalez, J. A. Abasy Atlas: a comprehensive inventory of systems, global network properties and systems-level elements across bacteria. Database (Oxford) 2016, doi:10.1093/database/baw089 (2016).

31 Camon, E. et al. The Gene Ontology Annotation (GOA) Database: sharing knowledge in Uniprot with Gene Ontology. Nucleic Acids Res 32, D262-266, doi:10.1093/nar/gkh021 (2004). 
32 Moretto, M. et al. COLOMBOS v3.0: leveraging gene expression compendia for crossspecies analyses. Nucleic Acids Res 44, D620-623, doi:10.1093/nar/gkv1251 (2016).

33 Clough, E. \& Barrett, T. The Gene Expression Omnibus Database. Methods Mol Biol 1418, 93-110, doi:10.1007/978-1-4939-3578-9_5 (2016).

34 Irizarry, R. A. et al. Summaries of Affymetrix GeneChip probe level data. Nucleic Acids Res 31, e15, doi:10.1093/nar/gng015 (2003).

35 Johnson, W. E., Li, C. \& Rabinovic, A. Adjusting batch effects in microarray expression data using empirical Bayes methods. Biostatistics 8, 118-127, doi:10.1093/biostatistics/kxj037 (2007).

36 Saito, T. \& Rehmsmeier, M. The precision-recall plot is more informative than the ROC plot when evaluating binary classifiers on imbalanced datasets. PLoS One 10, e0118432, doi:10.1371/journal.pone.0118432 (2015).

37 Siegenthaler, C. \& Gunawan, R. Assessment of network inference methods: how to cope with an underdetermined problem. PLoS One 9, e90481, doi:10.1371/journal.pone.0090481 (2014).

38 Campos, A. I. \& Freyre-Gonzalez, J. A. Evolutionary constraints on the complexity of genetic regulatory networks allow predictions of the total number of genetic interactions. Sci Rep 9, 3618, doi:10.1038/s41598-019-39866-z (2019).

39 Barabasi, A. L. \& Oltvai, Z. N. Network biology: understanding the cell's functional organization. Nat Rev Genet 5, 101-113, doi:10.1038/nrg1272 (2004).

40 Barabási, A.-L. \& Pósfai, M. Network Science. Edición: 1 edn, (Cambridge University Press, 2016).

41 Clauset, A., Shalizi, C. R. \& Newman, M. E. J. Power-Law Distributions in Empirical Data. SIAM Review 51, 661-703, doi:10.1137/070710111 (2009).

42 Khanin, R. \& Wit, E. How scale-free are biological networks. J Comput Biol 13, 810-818, doi:10.1089/cmb.2006.13.810 (2006).

43 Schieber, T. A. et al. Quantification of network structural dissimilarities. Nat Commun 8, 13928, doi:10.1038/ncomms13928 (2017).

44 Simpson, G. G. Mammals and the nature of continents. American Journal of Science 241, 131, doi:10.2475/ajs.241.1.1 (1943).

45 Freyre-Gonzalez, J. A., Trevino-Quintanilla, L. G., Valtierra-Gutierrez, I. A., Gutierrez-Rios, R. M. \& Alonso-Pavon, J. A. Prokaryotic regulatory systems biology: Common principles governing the functional architectures of Bacillus subtilis and Escherichia coli unveiled by the natural decomposition approach. J Biotechnol 161, 278-286, doi:10.1016/j.jbiotec.2012.03.028 (2012).

46 Chicco, D. \& Jurman, G. The advantages of the Matthews correlation coefficient (MCC) over F1 score and accuracy in binary classification evaluation. BMC Genomics 21, 6, doi:10.1186/s12864-019-6413-7 (2020).

47 Marbach, D. et al. Predictive regulatory models in Drosophila melanogaster by integrative inference of transcriptional networks. Genome Res 22, 1334-1349, doi:10.1101/gr.127191.111 (2012).

48 Lihu, A. \& Holban, S. A review of ensemble methods for de novo motif discovery in ChIPSeq data. Brief Bioinform 16, 964-973, doi:10.1093/bib/bbv022 (2015).

49 Liu, M. et al. Novel Two-Component System MacRS Is a Pleiotropic Regulator That Controls Multiple Morphogenic Membrane Protein Genes in Streptomyces coelicolor. Appl Environ Microbiol 85, doi:10.1128/AEM.02178-18 (2019).

50 Valdez, F., Gonzalez-Ceron, G., Kieser, H. M. \& Servi, N. G. L. The Streptomyces coelicolor A3(2) lipAR operon encodes an extracellular lipase and a new type of transcriptional 
regulator. Microbiology (Reading) 145 ( Pt 9), 2365-2374, doi:10.1099/00221287-145-92365 (1999).

51 Aigle, B., Wietzorrek, A., Takano, E. \& Bibb, M. J. A single amino acid substitution in region 1.2 of the principal sigma factor of Streptomyces coelicolor $A 3(2)$ results in pleiotropic loss of antibiotic production. Mol Microbiol 37, 995-1004, doi:10.1046/j.13652958.2000.02022.x (2000).

52 Li, L., Jiang, W. \& Lu, Y. A Novel Two-Component System, GluR-GluK, Involved in Glutamate Sensing and Uptake in Streptomyces coelicolor. J Bacteriol 199, doi:10.1128/JB.00097-17 (2017).

53 Mao, X. M. et al. Positive feedback regulation of stgR expression for secondary metabolism in Streptomyces coelicolor. J Bacteriol 195, 2072-2078, doi:10.1128/JB.00040-13 (2013).

54 Yang, H. et al. The tyrosine degradation gene hppD is transcriptionally activated by HpdA and repressed by HpdR in Streptomyces coelicolor, while hpdA is negatively autoregulated and repressed by HpdR. Mol Microbiol 65, 1064-1077, doi:10.1111/j.13652958.2007.05848.x (2007).

55 Urem, M. et al. OsdR of Streptomyces coelicolor and the Dormancy Regulator DevR of Mycobacterium tuberculosis Control Overlapping Regulons. mSystems 1, doi:10.1128/mSystems.00014-16 (2016).

56 Nguyen, K. T. et al. A central regulator of morphological differentiation in the multicellular bacterium Streptomyces coelicolor. Mol Microbiol 46, 1223-1238, doi:10.1046/j.13652958.2002.03255.x (2002).

57 Traag, B. A., Kelemen, G. H. \& Van Wezel, G. P. Transcription of the sporulation gene ssgA is activated by the IcIR-type regulator SsgR in a whi-independent manner in Streptomyces coelicolor A3(2). Mol Microbiol 53, 985-1000, doi:10.1111/j.1365-2958.2004.04186.x (2004).

58 Amin, R., Reuther, J., Bera, A., Wohlleben, W. \& Mast, Y. A novel GInR target gene, nnaR, is involved in nitrate/nitrite assimilation in Streptomyces coelicolor. Microbiology 158, 11721182, doi:10.1099/mic.0.054817-0 (2012).

59 Alkema, W. B., Lenhard, B. \& Wasserman, W. W. Regulog analysis: detection of conserved regulatory networks across bacteria: application to Staphylococcus aureus. Genome Res 14, 1362-1373, doi:10.1101/gr.2242604 (2004).

60 Emms, D. M. \& Kelly, S. OrthoFinder: phylogenetic orthology inference for comparative genomics. Genome Biol 20, 238, doi:10.1186/s13059-019-1832-y (2019).

61 Bailey, T. L. \& Elkan, C. Fitting a mixture model by expectation maximization to discover motifs in biopolymers. Proc Int Conf Intell Syst Mol Biol 2, 28-36 (1994).

62 Liu, X., Brutlag, D. L. \& Liu, J. S. BioProspector: discovering conserved DNA motifs in upstream regulatory regions of co-expressed genes. Pac Symp Biocomput, 127-138 (2001).

63 Liu, X. S., Brutlag, D. L. \& Liu, J. S. An algorithm for finding protein-DNA binding sites with applications to chromatin-immunoprecipitation microarray experiments. Nat Biotechnol 20, 835-839, doi:10.1038/nbt717 (2002).

64 Grant, C. E., Bailey, T. L. \& Noble, W. S. FIMO: scanning for occurrences of a given motif. Bioinformatics 27, 1017-1018, doi:10.1093/bioinformatics/btr064 (2011).

65 Gautier, L., Cope, L., Bolstad, B. M. \& Irizarry, R. A. affy--analysis of Affymetrix GeneChip data at the probe level. Bioinformatics 20, 307-315, doi:10.1093/bioinformatics/btg405 (2004).

66 Reese, S. E. et al. A new statistic for identifying batch effects in high-throughput genomic data that uses guided principal component analysis. Bioinformatics 29, 2877-2883, doi:10.1093/bioinformatics/btt480 (2013). 
67 Leek JT, J. W., Parker HS, Fertig EJ, Jaffe AE, Zhang Y, Storey JD, Torres LC. (R package version 3.38.0, 2020).

68 Faith, J. J. et al. Large-scale mapping and validation of Escherichia coli transcriptional regulation from a compendium of expression profiles. PLoS Biol 5, e8, doi:10.1371/journal.pbio.0050008 (2007).

69 Huynh-Thu, V. A., Irrthum, A., Wehenkel, L. \& Geurts, P. Inferring regulatory networks from expression data using tree-based methods. PLoS One 5, doi:10.1371/journal.pone.0012776 (2010).

70 Bonneau, R. et al. The Inferelator: an algorithm for learning parsimonious regulatory networks from systems-biology data sets de novo. Genome Biol 7, R36, doi:10.1186/gb2006-7-5-r36 (2006).

71 Meyer, P. E., Kontos, K., Lafitte, F. \& Bontempi, G. Information-theoretic inference of large transcriptional regulatory networks. EURASIP J Bioinform Syst Biol, 79879, doi:10.1155/2007/79879 (2007).

72 Haury, A. C., Mordelet, F., Vera-Licona, P. \& Vert, J. P. TIGRESS: Trustful Inference of Gene REgulation using Stability Selection. BMC Syst Biol 6, 145, doi:10.1186/1752-0509-6-145 (2012).

73 Nguyen, N. T. T. et al. RSAT 2018: regulatory sequence analysis tools 20th anniversary. Nucleic Acids Res 46, W209-W214, doi:10.1093/nar/gky317 (2018).

\section{Acknowledgments}

The authors thank to Yuko Makita and Prof. Kenta Nakai from the RIKEN Center for Sustainable Resource Science for kindly providing data from DBSCR. This work was supported by the Programa de Apoyo a Proyectos de Investigación e Innovación Tecnológica (PAPIIT-UNAM) [IN205918 and IN202421 to J.A.F.-G.]. A.Z.-A. received a doctoral scholarship [call 647 (2014)] from the Colombian Administrative Department of Science, Technology and Innovation (COLCIENCIAS). J.M.E.-R. is a doctoral student from Programa de Doctorado en Ciencias Biomédicas, Universidad Nacional Autónoma de México (UNAM); he received fellowship 959406 from CONACYT. We thank one reviewer for helpful and encouraging suggestions.

\section{Authors' contributions}

Conceptualization, J.A.F.G.; Methodology, A.Z.A., J.M.E.R., J.K.G.K., and J.A.F.G.; Software, A.Z.A., J.M.E.R., J.K.G.K. and J.A.F.G; Validation, A.Z.A., J.M.E.R., and J.A.F.G; Formal Analysis, A.Z.A., J.M.E.R., J.K.G.K., and J.A.F.G; Investigation, A.Z.A., J.M.E.R., J.K.G.K., and J.A.F.G; Resources, J.A.F.G.; Data Curation, A.Z.A.; Writing - Original Draft Preparation, A.Z.A., J.M.E.R, and J.K.G.K.; Writing - Review \& Editing, A.Z.A., J.M.E.R, and J.A.F.G; Visualization, J.M.E.R.; Supervision, J.A.F.G.; Project Administration, J.A.F.G.; Funding Acquisition, J.A.F.G.

\section{Additional Information}

\section{Competing interests}

The authors declare that they have no competing interests. 


\section{Supplementary Information}

- Supplementary File 1.docx: Word file with the Supplementary figures 1 - 14. NDA and structural analysis of the Curated_FL-DBSCR-RTB network. Supplementary methods and references.

- Supplementary File 2.xlsx: Excel file with Supplementary tables 1 - 10.

- Supplementary File 3.xlsx: Compressed folder with all the flat Files of the inferred networks.

\section{Figure legends}

Figure 1. a) Workflow of this work. The purple area covers the inference of the networks. b) Type of interactions contained in the networks. The green path connects to curated regulations supported by experimental evidence. The Strong network contains only the interactions that are supported by an experiment proving the transcription factor binds a DNA site near a target gene to regulate its transcription. Curated networks without the Strong label might contain indirect interactions, as they could be supported by non-directed experiments (such as gene knockout and its effect on genes transcription). The purple path connects to inferred interactions. Predictions based solely on binding sites predictions would be only inferring TF-DNA interactions. Predictions involving gene expression data might contain interactions with intermediates involved.

Figure 2. Interactions curated from literature for Streptomyces coelicolor A3(2). a) Number of publications per year and b) Number of interactions reported per year.

Figure 3. a) AUROC and AUPR for each of the methods and the community networks. b) Number of interactions statistically validated by TF

Figure 4. Network comparative by structural properties. a) Pearson correlation of the profile of structural properties listed in Supplementary Figure 12 . b) D-value from ${ }^{43}$ to identify network similarity.

Figure 5. Simpson index for NDA analysis for all curated and inferred networks. a) Global regulators. b) Modular genes. c) Intermodular genes. d) Basal machinery genes.

Figure 6. MCC for global regulators predicted by NDA for each of the curated and inferred networks. Scores $\geq 0.5$ are represented in white numbers and meta-curations are marked with an asterisk. a) Gold standard curated from reference ${ }^{28}$. b) Gold standard curated from independent publications (Supplementary Table 4).

Figure 7. Conservation of the systems-level components between S. coelicolor and C. glutamicum. a) NDA classification for the GRN-wide orthologs in their corresponding organism. Total matrix sum to 1 . Most of the GRN-wide orthologs are classified as basal machinery in both organisms b) Overlapping of the NDA classes between the two networks with reference to the smallest set. Each cell can range between 0 and 1, where 1 means one class is a subset of another, and 0 means there is no overlap at all. c) Similarities between the two organisms highlight the size difference between the datasets. The color of the inner circle sections represents the NDA classes and ribbons colors represent the $S$. coelicolor NDA classes. Numbers represent the genes for each class and organism. Gray ribbons are the widest ones, representing that all of the basal machinery of $S$. coelicolor with 1:1 orthology relationship with C. glutamicum is classified either as basal machinery 
or modular genes in $C$. glutamicum. This suggests that multiple basal machinery genes could be reclassified as modular components in more complete reconstructions of the $S$. coelicolor network.

\section{Tables}

Table 1. Description of networks used in this work.

\begin{tabular}{|c|c|c|c|c|}
\hline Network & Abasy ID & Genes & Interactions & Description \\
\hline Curated_RTB & 100226_v2015_sRTB13 & 311 & 330 & $\begin{array}{l}\text { Network from RegTransBase } \\
\text { database }\end{array}$ \\
\hline Curated_DBSCR & 100226_v2015_sDBSCR15 & 273 & 341 & $\begin{array}{l}\text { Network from Database of } \\
\text { transcriptional regulation } \\
\text { in Streptomyces coelicolor and } \\
\text { its closest relatives. }\end{array}$ \\
\hline Curated_DBSCR(S) & 100226_v2015_sDBSCR15_eStrong & 112 & 115 & $\begin{array}{l}\text { Filtration of interactions with } \\
\text { strong evidence from the } \\
\text { DBSCR network. }\end{array}$ \\
\hline Curated_FL & 100226_v2019_sA22 & 5331 & 9454 & $\begin{array}{l}\text { Network from the collection } \\
\text { and curation performed for } \\
\text { this work. }\end{array}$ \\
\hline Curated_FL(cS) & Not Reported & 347 & 438 & $\begin{array}{l}\text { Filtration of interactions with } \\
\text { strong evidence from the } \mathrm{FL} \\
\text { network ( } c S=\text { curated strong) }\end{array}$ \\
\hline Curated_FL(S) & 100226_v2019_sA22_eStrong & 396 & 493 & $\begin{array}{l}\text { Filtration of interactions with } \\
\text { strong evidence from the FL } \\
\text { network along with statistically } \\
\text { validated interactions. }\end{array}$ \\
\hline $\begin{array}{l}\text { Curated_FL-DBSCR- } \\
\text { RTB }\end{array}$ & 100226_v2019_sA22-DBSCR15-RTB13 & 5386 & 9707 & $\begin{array}{l}\text { Meta-curation of RTB, DBSCR } \\
\text { and FL networks. }\end{array}$ \\
\hline $\begin{array}{l}\text { Curated_FL(cS)- } \\
\operatorname{DBSCR}(S)\end{array}$ & Not Reported & 387 & 480 & $\begin{array}{l}\text { Filtration of interactions with } \\
\text { strong evidence from the } \\
\text { meta-curated network. }\end{array}$ \\
\hline $\begin{array}{l}\text { Curated_FL(S)- } \\
\operatorname{DBSCR}(S)\end{array}$ & $\begin{array}{l}\text { 100226_v2019_sA22- } \\
\text { DBSCR15_eStrong }\end{array}$ & 435 & 534 & $\begin{array}{l}\text { Filtration of interactions with } \\
\text { strong evidence from meta- } \\
\text { curated networks along with } \\
\text { statistically validated } \\
\text { interactions. }\end{array}$ \\
\hline Inferred_BS & Available as a Supplementary File 3 & 6263 & 23908 & $\begin{array}{l}\text { Inferred GRN from binding } \\
\text { sites prediction. }\end{array}$ \\
\hline Inferred_Exp & Available as a Supplementary File 3 & 4739 & 23908 & $\begin{array}{l}\text { Inferred GRN from } \\
\text { transcriptomic data. }\end{array}$ \\
\hline Inferred_BS-Exp & Available as a Supplementary File 3 & 4763 & 23908 & $\begin{array}{l}\text { Community network from } \\
\text { Inferred_BS and Inferred_Exp. }\end{array}$ \\
\hline Inferred_All & Available as a Supplementary File 3 & 3804 & 23908 & $\begin{array}{l}\text { Community network from all } \\
\text { the inference methods. }\end{array}$ \\
\hline
\end{tabular}

Table 2. Network properties for inferred networks

\begin{tabular}{l|l|l|l|l|l} 
Property & Inferred_BS & Inferred_Exp & Inferred_BS-Exp & Inferred_All & Curated_FL-
\end{tabular}


bioRxiv preprint doi: https://doi.org/10.1101/2021.1126.470080. this version posted December 4, 2021. The copyright holder for this preprint (which was not certified by peer review) is the author/funder, who has granted bioRxiv a license to display the preprint in perpetuity. It is made available under aCC-BY 4.0 International license.

\begin{tabular}{|l|c|c|c|c|c|}
\hline & & & & & DBSCR-RTB \\
\hline Number of nodes $(\mathrm{N})$ & 6263 & 4739 & 4763 & 3804 & 5386 \\
\hline $\ln (\ln (N))$ & 2.17 & 2.14 & 2.14 & 2.11 & 2.15 \\
\hline $\begin{array}{l}\text { Average shortest } \\
\text { path length }\end{array}$ & 2.86 & 3.38 & 3.38 & 3.11 & 2.84 \\
\hline $\begin{array}{l}\text { Average clustering } \\
\text { coefficient }\end{array}$ & 0.213 & 0.385 & 0.385 & 0.470 & 0.182 \\
\hline$\alpha(P(k))$ & 1.861 & 1.952 & 1.955 & 1.968 & 1.742 \\
\hline $\left.\mathrm{R}^{2}{ }_{\text {ddj }}(k)\right)$ & 0.87 & 0.92 & 0.92 & 0.91 & 0.84 \\
\hline$\alpha(C(k))$ & 0.924 & 0.767 & 0.742 & 0.729 & 1.142 \\
\hline $\mathrm{R}^{2}{ }_{\text {adj }}$ & 0.79 & 0.58 & 0.54 & 0.68 & 0.89 \\
\hline
\end{tabular}




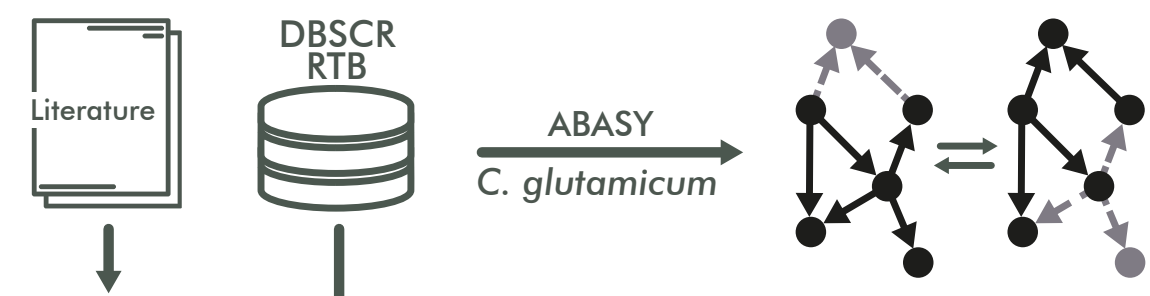

Evidence

Curated 9186

TFs

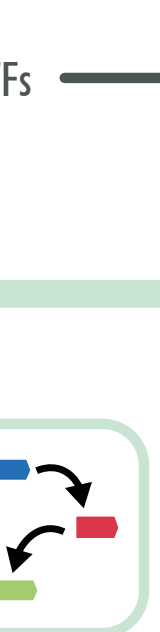

100226_v2019_sFL-DBSCR15-RTB13 experimentally supported interactions

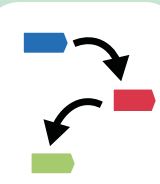

ם

100226_v2019_sFL-DBSCR15_eStrong
Gold standard

Cross-validation validated

S. coelicolor

networks

Q

D

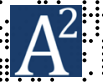

口

Best tool

1

Assessment

Inferred networks

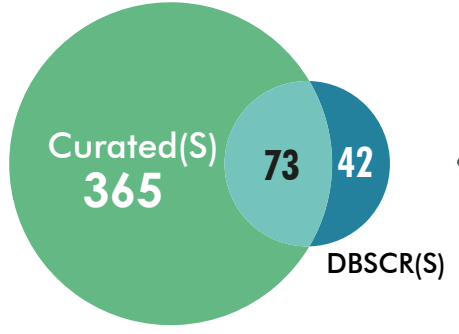

Gene expression

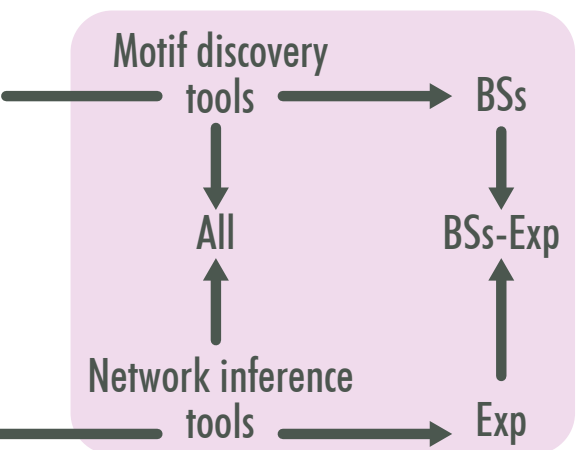

Global TFs

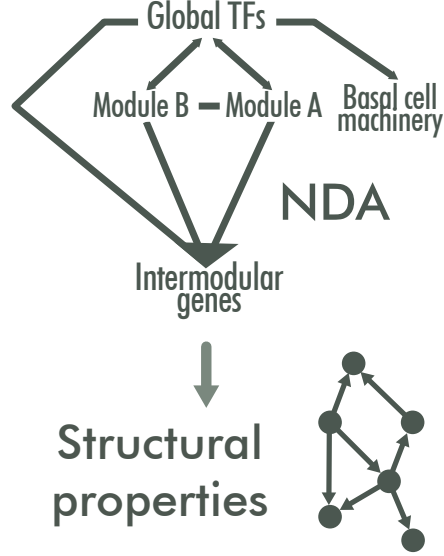

Gene or TF of interest
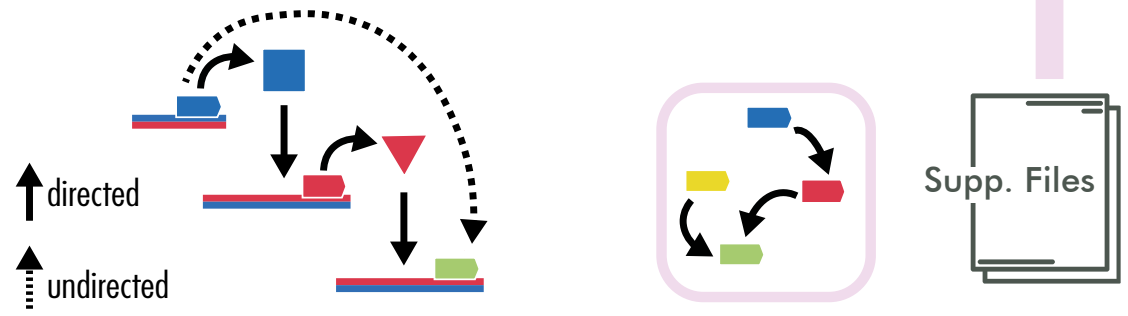

Inferred_BS
Inferred_BS-Exp Inferred_Exp Inferred_All

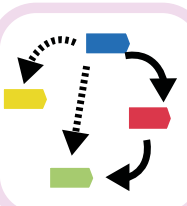




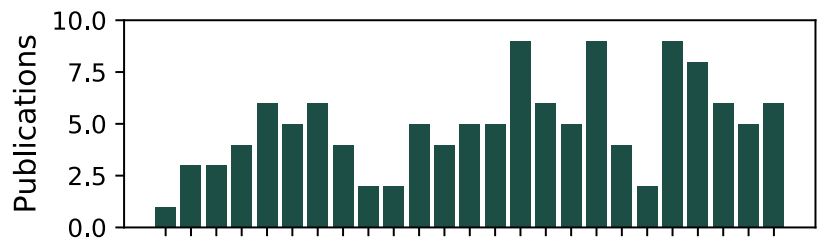

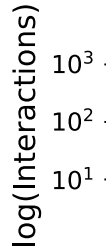

ONN बढंब ब ब ब ब

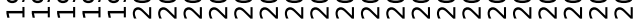
Year 


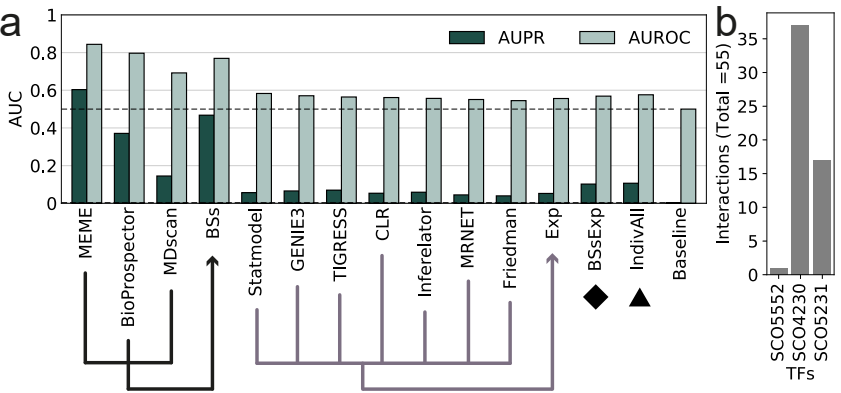


red All

Inferred_BS-Exp

Inferred_Exp

Curated_DBSCR(S)

Curated_RTB

Curated_DBSCR

Curated_FL(S)

Curated_FL(S)-DBSCR(S)

Inferred_BS —

Curated FL

Curated_FL-DBSCR-RTB

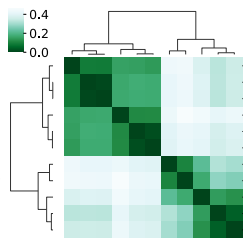

- Inferred_All «

- Inferred_BS-Exp —

- Inferred_Exp

- Inferred_BS

- Curated_FL

- Curated_FL-DBSCR-RTB

- Curated_DBSCR(S)

- Curated_RTB

- Curated_DBSCR

- Curated_FL(S)

- Curated_FL(S)-DBSCR(S) 
Coverage

\begin{tabular}{|c|c|c|c|c|c|}
\hline & & & & & \\
\hline Curated RTB - & 0.22 & 0.37 & 0.28 & 0.037 & 0.014 \\
\hline Curated_DBSSCR- & 0.27 & 0.46 & 0.41 & 0.033 & 0.014 \\
\hline Curated FL- & 0.49 & 0.65 & 0.77 & 0.64 & \\
\hline Curated_FL-DBSCR-RTB - & 0.47 & 0.65 & 0.76 & 0.65 & 0.42 \\
\hline Curated_DBSCR(S) & 0.56 & 0.54 & 0.61 & 0.013 & 0.0048 \\
\hline Curated_FL(S) - & 0.57 & 0.55 & 0.62 & 0.048 & 0.021 \\
\hline${ }^{*}$ Curated_FL(S)-DBSCR(S) - & 0.49 & 0.56 & 0.58 & 0.052 & 0.022 \\
\hline & 0.39 & 0.29 & 0.41 & 0.75 & \\
\hline Inferred_BS-Exp & 0.38 & 0.39 & 0.49 & 0.57 & 1 \\
\hline Inferred_EX & 0.38 & 0.39 & 0.49 & 0.57 & 1 \\
\hline Inferred_A & 0.35 & 0.3 & 0.42 & 0.46 & 1 \\
\hline & Reviewa $^{a}$ & Independent ${ }^{\mathrm{b}}$ & Both & Genomic & Interact \\
\hline
\end{tabular}




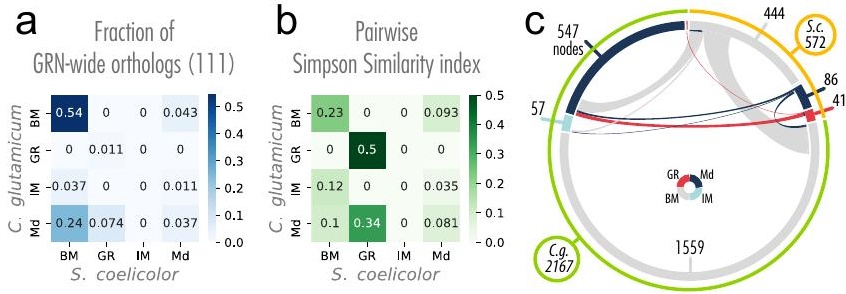

